\title{
ÓXIDOS DE COLESTEROL EM PESCADO*
}

FABIANA RODRIGUES DE OLIVEIRA**

GISELDA MACENA LIRA***

Em algumas espécies, o pescado apresenta ácidos graxos com alto grau de insaturação em sua fração lipídica, com níveis elevados de colesterol. Esse fator, associado a procedimentos tecnológicos favorece a oxidação do colesterol. Os óxidos de colesterol são biologicamente ativos, capazes de desencadear processos citotóxicos, aterogênicos, mutagênicos e carcinogênicos. Por essas razões, a preocupação com o fenômeno da oxidação tem aumentado consideravelmente. Neste trabalho de revisão foram abordadas questões relativas aos óxidos de colesterol, sua formação em alimentos, ocorrência em pescado, identificação, quantificação e efeitos biológicos. O estudo revelou que os óxidos de colesterol mais comuns em alimentos, incluindo os mais citotóxicos e aterogênicos, são também encontrados nesses produtos.

PALAVRAS-CHAVE: COLESTEROL; OXIDAÇÃO; ÓXIDOS DE COLESTEROL; PESCADO.

* Elaborado a partir da dissertação de mestrado em Nutrição da primeira autora.

** Mestre em Nutrição, Faculdade de Nutrição, Universidade Federal de Alagoas, Maceió, AL, Brasil (e-mail: lifagabi@ig.com.br).

*** Doutora em Ciências dos Alimentos, Faculdade de Nutrição, Universidade Federal de Alagoas, Maceió, AL, Brasil (e-mail: gmlira@superig.com.br). 


\section{INTRODUÇÃO}

Estudos demonstram que a oxidação de lipídios é uma das principais causas da deterioração de alimentos (LUZIA, 2000) e que se constitui em potencial fator de risco para a saúde (BAGGIO, 2004).

A extensão da oxidação lipídica depende de mecanismos reacionais diversos e extremamente complexos, que estão relacionados com o tipo de estrutura dos lipídios e o meio em que se encontram (SILVA, BORGES e FERREIRA, 1999).

Os ácidos graxos poli-insaturados são muito susceptíveis à oxidação, sendo facilmente incorporados na reação em cadeia da peroxidação lipídica, com formação de radicais livres e hidroperóxidos (SAMPAIO, 2004). Segundo SMITH (1987), os hidroperóxidos são os compostos que iniciam mais uma importante reação de oxidação, a do colesterol.

O colesterol, amplamente distribuído em alimentos de origem animal, rapidamente submetese à oxidação na presença do ar e sob diferentes condições (CHEN e CHEN, 1994). Os derivados da sua oxidação são óxidos biologicamente ativos de interesse, tanto tecnológico como clínico pela relação com processos citotóxicos, aterogênicos, mutagênicos e cancerígenos, além de outros efeitos deletérios (MORALES-AIZPURÚA e TENUTA-FILHO, 2005).

Já foram identificados mais de 80 produtos da oxidação do colesterol. Os mais comuns em alimentos são: 7-cetocolesterol (7-Ceto), 20-hidroxicolesterol (20-OH), 25-hidroxicolesterol (25-OH),

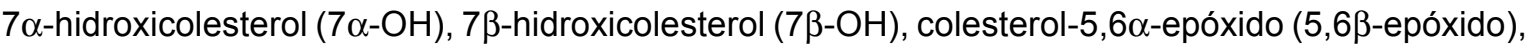

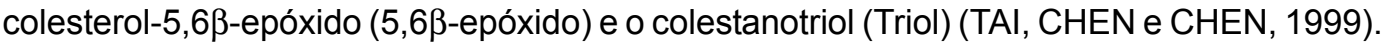

Dentre os alimentos de origem animal, o pescado está entre os mais propensos à deterioração oxidativa do colesterol devido à alta proporção de ácidos graxos poli-insaturados em sua fração lipídica e aos níveis relativamente elevados de colesterol de algumas espécies (JOHNSTON et al., 1983). $O$ tipo de processamento tecnológico e as condições de armazenamento também contribuem para que o pescado seja susceptível à presença de quantidades significativas de óxidos de colesterol (OsC) (ECHARTE et al., 2005). No entanto, apesar do relevante papel na alimentação (MUSTAFAe MEDEIROS, 1985), o pescado tem sido pouco estudado nesse sentido (MOURA e TENUTA-FILHO, 2002).

Com base no exposto, e considerando principalmente a preocupação crescente com o fenômeno da oxidação (SILVA, BORGES e FERREIRA, 1999), neste trabalho de revisão foram abordadas questões relativas aos óxidos de colesterol ( $\mathrm{OsC}$ ), sua formação, identificação, quantificação e efeitos biológicos, enfocando aspectos da oxidação do colesterol em pescado.

\section{COLESTEROL, COMPOSTO SUSCEPTÍVEL À OXIDAÇÃO}

O colesterol é o mais importante e abundante dos esteroides, lipídios cíclicos de grandes dimensões. Presente nas células de origem animal, compõe a estrutura das membranas celulares, sendo precursor para a síntese de substâncias de importância vital como a vitamina D3, os sais biliares e hormônios (estrógenos, aldosterona e cortisol, entre outros) (HARPER, RODWELL e MAYES, 1982). É encontrado nos sistemas biológicos e nos alimentos nas formas livre e esterificada. Segundo MASORO (1968), em tecidos e fluidos do fígado, córtex adrenal, plasma e linfa de mamíferos, o colesterol está principalmente na forma esterificada, enquanto na massa muscular esquelética encontrase predominantemente livre.

Como composto heterogêneo, da classe dos chamados terpenoides (CONN e STUMPF, 1975), o colesterol apresenta sistema de quatro anéis condensados que formam estrutura complexa chamada ciclopentanofenantreno (HOFFMANN, 1989). Por essa estrutura, que contém uma dupla ligação entre os carbonos C-5 e C-6 do anel B (MAERKER, 1987), o colesterol é caracterizado como lipídio insaturado susceptível à oxidação (SMITH, 1981 apud SMITH, 1996) e origina uma série de produtos oxidados com estruturas semelhantes, os OsC, que apresentam como grupos funcionais hidroxilas, cetona e epóxido adicionados no núcleo esterol e/ou na cadeia lateral da molécula (BAGGIO, 2004). 
A presença do grupo hidroxila na molécula de colesterol, $\beta$-orientado ligado ao carbono $C$ - 3 do anel A (MAERKER, 1987), confere-lhe alguma afinidade pelo meio aquoso. O restante da molécula, no entanto, é formado por anéis de hidrocarboneto, tornando-o praticamente insolúvel em água (SAMPAIO, 2004). Esse caráter anfipático do colesterol permite-lhe interagir com outros lipídios na interfase óleoágua, o que também facilita a sua oxidação (GUARDIOLA et al., 1995b).

\subsection{FORMAÇÃO DOS ÓXIDOS DE COLESTEROL (OsC)}

A oxidação do colesterol pode ocorrer por mecanismos enzimáticos e não enzimáticos (GUARDIOLA et al., 1995b). Em sistemas biológicos, os OsC são formados por via enzimática, basicamente no fígado e nos tecidos geradores de hormônios esteroides (córtex supra-renal, gônadas) (SMITH, 1996). Nos alimentos, a oxidação ocorre por mecanismos não enzimáticos: autoxidação, peroxidação lipídica e oxidação fotoquímica (MORALES-AIZPURÚA e TENUTA-FILHO, 2002).

A autoxidação inicia-se pela formação de um radical no carbono $C-7$ do anel $B$, que reage com o oxigênio molecular triplete $\left({ }^{3} \mathrm{O}_{2}\right)$, conduzindo à formação de dois 7-hidroperóxidos ( $\alpha$ e $\beta$ ) (PENG et al., 1979). Esses hidroperóxidos são termicamente instáveis, decompondo-se em $7 \alpha-\mathrm{OH}, 7 \beta-\mathrm{OH}$ e 7 Ceto. $\mathrm{O} 7 \alpha-\mathrm{OH}$ e o $7 \beta-\mathrm{OH}$ encontram-se em equilíbrio, mas com tendência à predominância da forma $\beta$ (SMITH apud CRASTES DE PAULET, DOUSTE-BLAZY e PAOLETTI, 1990), devido à estabilidade da conformação equatorial em relação à axial (SMITH, 1981 apud SMITH, 1996). O 7-Ceto pode decomporse em 3,5-colestadien-7-ona em meio ácido e sob aquecimento (SMITH apud CRASTES DE PAULET, DOUSTE-BLAZY e PAOLETTI, 1990). SMITH (1981) apud SMITH (1996) relatou que secundariamente à formação dos 7-hidroperóxidos ocorre também a oxidação nos carbonos C-5 e C-6. O 5,6 $\alpha$-epóxido e o 5,6ß-epóxido são os produtos do ataque à dupla ligação do colesterol (MAERKER, 1987). Sua hidratação leva à formação do Triol, podendo decompor-se, ainda, em 3 $\beta, 5$-di-hidroxi-5 $\alpha$-colestano-6ona (SMITH, 1981 apud SMITH, 1996).

Por autoxidação do colesterol podem ser formados outros radicais peroxilas e seus correspondentes hidroperóxidos, como os derivados hidroxilados $(20-\mathrm{OH}, 24-\mathrm{OH}, 25-\mathrm{OH}, 26-\mathrm{OH}$ e $27-$ $\mathrm{OH})$ na cadeia lateral por reações de transferência de radicais, destacando-se os formados nos carbonos 20 e 25 (20-OH e 25-OH) (GUARDIOLA et al., 1995b).

Quando o colesterol está esterificado, a autoxidação envolve o mesmo processo que ocorre no colesterol livre, mas em velocidade diferente (SMITH, 1987). O 7 $\alpha-\mathrm{OH}, 7 \beta-\mathrm{OH}, 7-C e t o, 5,6 \alpha$-epóxido e o 5,6ß-epóxido são os produtos da autoxidação do colesterol éster (KORAHANI, BASCOUL e CRASTED DE PAULET, 1982).

Segundo GUARDIOLA et al. (1995b), os mesmos OsC da autoxidação no anel B do colesterol formam-se via peroxidação lipídica: $7 \alpha-\mathrm{OH}, 7 \beta-\mathrm{OH}, 7$-Ceto, 5,6 $\alpha$-epóxido, 5,6 $\beta$-epóxido e Triol. Por esse mecanismo, o colesterol é oxidado por hidroperóxidos ou peróxidos cíclicos, derivados da oxidação (com intervenção enzimática) de outros lipídios.

$\mathrm{Na}$ oxidação fotoquímica, o responsável pelo início do processo é o oxigênio singlete $\left({ }^{1} \mathrm{O}_{2}\right)$, forma mais ativa do oxigênio, que se origina da transferência da energia absorvida por sensitizadores (como clorofila e hematoporfirina) para o oxigênio triplete $\left({ }^{3} \mathrm{O}_{2}\right)$ (GUARDIOLA et al., 1995b; NIELSEN et al., 1996). É dessa reação que derivam os hidroperóxidos geradores de $7 \alpha-\mathrm{OH}$ e 7-Ceto (GUARDIOLA et al., 1995b).

\subsection{FATORES QUE AFETAM A OXIDAÇÃO DO COLESTEROL}

O colesterol é instável sob grande variedade de condições, como luz, calor, radiação, presença de radicais livres, oxigênio e metais de transição (PANIANGVAIT et al., 1995), parâmetros que têm sido estudados como fatores influenciadores da sua oxidação em alimentos (SAMPAIO, 2004).

Caracterizada como sistema dependente (MORALES-AIZPURÚA e TENUTA-FILHO, 2002), a oxidação do colesterol em alimentos depende ainda da atuação isolada ou concomitante de outras 
variáveis, incluindo as próprias características do alimento: conteúdo de água, $\mathrm{pH}$, efeito tamponante, concentração e formas física e química do colesterol, tipo de ácido graxo presente, presença de antioxidantes ou pró-oxidantes, e as interações entre seus componentes e produtos de decomposição durante o processamento e/ou armazenamento (SMITH, 1987; PANIANGVAIT et al., 1995). No entanto, pesquisas têm comprovado que a formação dos OsC em alimentos é especialmente determinada pela combinação de três fatores: temperatura elevada, disponibilidade de oxigênio e de ácidos graxos poliinsaturados (TAI, CHEN e CHEN, 1999).

Alimentos submetidos a processos tecnológicos que envolvam exposições ao calor apresentam grande potencial para a oxidação, merecendo, portanto, atenção especial (MOURA, 1999). Em seu processamento, alguns pescados são expostos drasticamente a temperaturas elevadas, mas poucos são os dados sobre a ocorrência de OsC nesses produtos (SEBEDIO et al., 1993).

OSADA et al. (1993) não encontraram OsC em estudo com pescado cru, mas quando submetidos à secagem ao sol verificaram concentrações significativas desses óxidos em sardinhas e lulas (287 ppm e 146 ppm, respectivamente). Valores menores foram descobertos por KAO e HWANGSUN (1997) analisando OsC em lulas desidratadas, que quando assadas a $200^{\circ} \mathrm{C}$ por 10 minutos apresentaram diminuição do colesterol de 7300 ppm para 6020 ppm e aumento dos OsC de 12,07 ppm para $43,46 \mathrm{ppm}$.

A forma de exposição ao calor, segundo MORGAN e ARMSTRONG (1992), constitui fator importante na oxidação do colesterol, sendo maior a formação dos OsC em alimentos submetidos a aquecimento direto.

Em pesquisa de OsC em anchovas cozidas e grelhadas, OHSHIMA et al. (1996) constataram que os níveis aumentaram quando grelhadas a $220^{\circ} \mathrm{C}$ por 6 minutos. SAMPAIO (2004) também reportou altas quantidades de $\mathrm{OsC}$ em camarão salgado-seco, atribuídas à associação dos níveis elevados de colesterol do camarão, $90 \mathrm{mg} / 100 \mathrm{~g}$ a $200 \mathrm{mg} / 100 \mathrm{~g}$ (JOHNSTON et al., 1983) com condições inadequadas de armazenamento e processamento, dentre as quais a exposição à alta temperatura durante a secagem ao sol.

O sal também se torna forte pró-oxidante das gorduras em associação com o calor, ativando a lipoxidase do músculo (PARDI et al., 2001).

Estudando a estabilidade do colesterol durante o aquecimento, OSADA et al. (1993) constataram que a oxidação é ainda influenciada pelo tempo de aquecimento: a $100^{\circ} \mathrm{C}$ por 24 horas foram formados seis tipos de óxidos $\left(7 \alpha-\mathrm{OH}, 7 \beta-\mathrm{OH}, 7-\mathrm{Ceto}, 5,6 \alpha\right.$-epóxido e Triol), mas a $200^{\circ} \mathrm{C}$ por 6 horas houve destruição completa do colesterol e de todos os óxidos formados.

Investigando o efeito do tempo e da temperatura de processamento sobre a oxidação do colesterol em alimentos, CHEN, WANG e CHEN (1998) comprovaram a influência do binômio tempo/ temperatura sobre a oxidação. Porém, observaram que a concentração de $\mathrm{OsC}$ diminuiu com a redução do tempo de aquecimento.

Dentre os fatores mais importantes para a oxidação do colesterol em alimentos, a presença de ácidos graxos poli-insaturados revelou a maior correlação com o perfil dos óxidos formados e suas quantidades relativas (PANIANGVAIT et al., 1995).

Os ácidos graxos poli-insaturados são mais propensos à peroxidação lipídica pela maior suscetibilidade dos átomos de hidrogênio bi-alílicos do grupo metileno à abstração por radicais oxidáveis, que os hidrogênios metilênicos dos lipídios saturados (DORMANDY apud GUTTERIDGE e HALLIWELL, 1994). Da oxidação dos ácidos graxos poli-insaturados resultam os hidroperóxidos que iniciam a oxidação do colesterol, podendo aumentá-la sinergicamente (SMITH, 1987).

Ll et al. (1996) estudaram a influência da composição de ácidos graxos sobre a oxidação do colesterol em óleos de pescado, linhaça, girassol e palma, durante estocagem e aquecimento. Constataram que no óleo de peixe formaram-se mais $\mathrm{OsC}$, e que após 35 dias de armazenamento a concentração era três vezes maior que nos óleos vegetais. A maior proporção de $\mathrm{OsC}$ no óleo de peixe deve-se aos seus níveis mais elevados de ácidos graxos insaturados, em especial os poli-insaturados ômega-3 (n3). Mas a presença de antioxidantes nos óleos vegetais, como o tocoferol, também pode ter inibido a oxidação do colesterol (NAWAR apud FENNEMA, 1996). 
Segundo BADOLATO et al. (1994), o óleo de peixe contém grande variedade de ácidos graxos com 20 a 22 átomos de carbono, altamente insaturados, destacando-se o eicosapentaenoico (C20:5 - EPA) e o docosahexaenoico (C22:6 - DHA) da série ômega-3, os quais não ocorrem em outros animais além de traços.

\section{OCORRÊNCIA DE ÓXIDOS DE COLESTEROL EM PESCADO}

Os OsC encontrados em pescado são os derivados predominantemente da oxidação no anel B, com destaque para o $7 \alpha-\mathrm{OH}, 7 \beta-\mathrm{OH}$ e o 7-Ceto (CHEN e CHEN, 1994; OSADA et al., 1993; OHSHIMA, LI e KOIZUMI, 1993). Entretanto, segundo OHSHIMA et al. (1996), outros óxidos também são encontrados em menor proporção como o 25-OH e o Triol.

O 7ß-OH foi o óxido identificado em maior quantidade por SAMPAIO (2004) no camarão salgadoseco, $5,44 \mu \mathrm{g} / \mathrm{g}$ a 42,86 $\mu \mathrm{g} / \mathrm{g}$, e por outros autores em trabalhos com pescado (OHSHIMA et al., 1996; OHSHIMA, LI e KOIZUMI, 1993). Em peixes secos, CHEN e YEH (1994) encontraram o $7 \alpha-\mathrm{OH}, 7 \beta-$

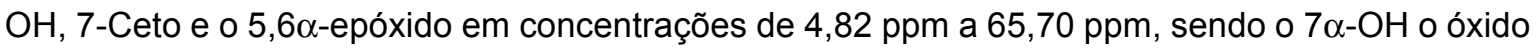
predominante. Em análise de amostras comerciais de pescado marinho, OHSHIMA, LI e KOIZUMI (1993) consideraram o $7 \beta-\mathrm{OH}$ e o 7 -Ceto como os óxidos mais importantes.

O 7-Ceto tem sido utilizado como indicador da oxidação do colesterol em alimentos por ser produzido em maior quantidade e nos estágios iniciais do processo oxidativo (RODRIGUEZ-ESTRADA et al., 1997).

Em atum enlatado, ZUNIN, BOGGIAe EVANGELISTI (2001) detectaram 0,3 $\mu \mathrm{g} / \mathrm{g}$ a $3,0 \mu \mathrm{g} / \mathrm{g}$ de OsC, sendo o 7-Ceto o óxido de maior formação. Segundo tais autores, essa taxa de oxidação poderia ter sido maior se em seu processamento o atum não sofresse redução significativa do teor de lipídios. O conteúdo de lipídios dos alimentos tem grande relevância no processo de oxidação do colesterol, sendo menor a produção de óxidos em alimentos com baixo teor de gordura (OHSHIMA, LI e KOIZUMI, 1993). SHOZEN et al. (1995) analisaram pescado marinho no Japão e relataram forte correlação $\left(r^{2}=\right.$ 0,98 ) entre as concentrações de OsC e o conteúdo de lipídios dos peixes.

Peixes de qualquer espécie apresentam diferentes teores de gordura, classificando-se em magros e gordos conforme a idade, sexo, estado biológico, alimentação disponível, condições do habitat (temperatura da água) e o método de captura (SANCHEZ, 1989).

RODRIGUEZ-ESTRADA et al. (1997) também reportaram a presença de 7-Ceto em camarãorosa e em hambúrguer como indicativo da ocorrência de outros óxidos, produzidos na oxidação do colesterol.

Entre os OsC encontrados em pescado, o 25-OH é um dos que ocorre em maior concentração em alimentos (GUARDIOLA et al., 1995b), sendo considerado, com o Triol, os mais citotóxicos e aterogênicos (PENG, HU e MORIN, 1991).

KAO e HWANG-SUN (1997), analisando lulas desidratadas, encontraram o $7 \alpha-\mathrm{OH}, 7 \beta-\mathrm{OH}, 7-$

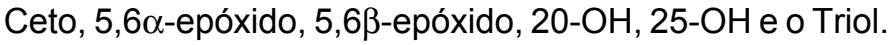

O 25-OH e o Triol também foram detectados por OHSHIMA, LI e KOIZUMI (1993) em espécie de camarão cozido-seco (Sergetes lucens). Esses mesmos óxidos foram encontrados por OHSHIMA et al. (1996) em estudo com anchovas cozidas e grelhadas.

Novos OsC foram descobertos em amostras de peixe, em estudo comparativo de detectores de produtos da oxidação do colesterol realizado por SALDANHA et al. (2006): 19-hidroxicolesterol, 22 "R"-hidroxicolesterol, 22 "S"-hidroxicolesterol, 24 "S"-hidroxicolesterol e 25 "R"-hidroxicolesterol. O 19-hidroxicolesterol normalmente não está presente em alimentos, razão pela qual é usado como padrão (amostra branca) na identificação de OsC. Sua presença, como a dos demais óxidos recém-descobertos em peixes, provavelmente está associada ao metabolismo dos animais (SALDANHA et al., 2006). 


\section{IDENTIFICAÇÃO E QUANTIFICAÇÃO DOS ÓXIDOS DE COLESTEROL}

Os avanços alcançados na identificação e quantificação de OsC em alimentos têm sido possíveis pelo desenvolvimento de técnicas de alta precisão (VALENZUELA, SANHUEZA e NIETO, 2002). As análises são fortemente influenciadas pela estrutura química complexa desses óxidos, que apresentam grupos funcionais que lhes confere diferentes polaridades e propriedades, e pela complexidade da fração lipídica de certos alimentos (GUARDIOLA et al., 2004; GUARDIOLA et al., 1995a).

A fase mais crítica para o procedimento analítico é a de extração e purificação das frações de OsC, processo que envolve saponificação e/ou cromatografia do extrato lipídico (GUARDIOLA et al., 1995a). Em alimentos com baixos níveis de OsC, a quantificação dos mesmos torna-se problema analítico, uma vez que a extração e a separação desses compostos sofrem frequentemente a influência de interferentes, como o próprio colesterol e outros esterois, os triacilglicerois e os fosfolipídeos (McCLUSKEY e DEVERY, 1993).

A determinação dos OsC pode ser realizada mediante várias metodologias, sendo as mais utilizadas a cromatografia a gás (CG) e a cromatografia a líquido de alta eficiência (CLAE) (McCLUSKEY e DEVERY, 1993).

Apesar de eficiente, a CG pode destruir termicamente o colesterol e os hidroperóxidos levando à formação de artefatos (GUARDIOLA et al., 2004). Isso requer derivação adicional, além da saponificação do extrato lipídico e subsequente extração da fração insaponificável que contém os óxidos, e torna a recuperação difícil (CARERI et al., 1998). Associada à espectrometria de massa (CG-MS), a CG tem sido apontada como a técnica mais eficaz para a confirmação dos OsC (OSADA et al., 1993). Como consequência das limitações da CG, a CLAE tornou-se a técnica escolhida (CHEN e CHEN, 1994; CARERI et al., 1998), principalmente porque simplifica os procedimentos de quantificação e diminui o número de artefatos (BAGGIO, 2004). Por essa técnica, numerosos sistemas de detecção têm sido usados para determinar OsC, destacando-se a espectrofotometria com detector de ultravioleta (UV), índice de refração (IR) e a espectrometria de massa (EM) (GUARDIOLA et al., 2004).

A determinação simultânea dos OsC por CLAE não é fácil, considerando que muitos deles têm estruturas semelhantes e a ausência de grupos cromóforos adequados para facilitar a detecção pelos métodos convencionais. Além disso, na maioria dos casos, estão em quantidades pequenas para a sensibilidade do detector. O uso da CLAE com a espectrometria de massa (CLAE-APCI-EM) é o sistema que tem permitido o aperfeiçoamento das possibilidades analíticas, principalmente dos compostos termolábeis, aumentando a sensibilidade (CARERI et al., 1998).

O detector ultravioleta (UV) mostra alta sensibilidade e especificidade, mas é limitado devido à absorção fraca e não específica na luz ultravioleta de alguns óxidos, como o 5,6 $\alpha$-epóxido, 5,6 $\beta$ epóxido e o Triol (GUARDIOLA et al., 2004; SMITH, 1993), exigindo determinações mais complexas. O IR, por sua vez, é de uso universal, podendo ser empregado com qualquer tipo de solvente sob condições isocráticas. Permite a detecção de todos os $\mathrm{OsC}$, mas apresenta menor sensibilidade que o UV (GUARDIOLA et al., 2004; CHEN, CHIU e CHEN, 1994).

SALDANHA et al. (2006) compararam os detectores CLAE (UV, IR e APCI-EM) na determinação simultânea de 12 produtos da oxidação do colesterol em peixes. Relataram que não houve diferença significativa na quantificação dos compostos entre os detectores, mas que houve clara identificação de vários tipos de OsC por CLAE-APCI-EM. Os autores também reportaram a descoberta de OsC nunca antes identificados em peixes.

\section{EFEITOS BIOLÓGICOS DOS ÓXIDOS DE COLESTEROL}

A oxidação do colesterol tem merecido atenção especial em razão das possíveis implicações dos OsC sobre a saúde humana. Estudos realizados em modelos celulares e com animais 
têm demonstrado o potencial desses compostos na ocorrência de efeitos biológicos adversos (TAYLOR et al., 1979).

Vários OsC têm sido detectados em quantidades significativas em fluidos e tecidos humanos, derivados da ingestão de alimentos contendo colesterol oxidado, da oxidação de lipoproteínas ou ainda do catabolismo intracelular (PINCINATO, 2000).

Os óxidos comumente encontrados em alimentos, como o $7 \alpha-\mathrm{OH}, 7 \beta-\mathrm{OH}, 7-\mathrm{Ceto}, 5,6 \alpha-$ epóxido, 5,63-epóxido, 25-OH e o Triol são citotóxicos e aterogênicos em diferentes graus (BÖSINGER, LUF e BRANDL, 1993). O 25-OH e o Triol são considerados os mais citotóxicos, podendo inibir completamente o crescimento e o desenvolvimento celular (BAGGIO, 2004). Os OsC, tóxicos ao endotélio, podem destruir a integridade endotelial, resultando na alteração da permeabilidade vascular, passo inicial para o desenvolvimento da aterosclerose (PENG, HU e MORIN, 1991).

Há muitas décadas é estimada forte correlação positiva entre a hipercolesterolemia e a aterogênese. No entanto, estudos têm sugerido que os OsC desempenham maior papel na formação de placas ateroscleróticas do que o próprio colesterol (TSAl et al., 1980).

ADDIS et al. (1995) apud ECHARTE, ZULET e ASTIASARÁN (2001) relataram que ainda não há evidências diretas em humanos de que a oxidação do colesterol contribua para a aterogênese, mas a presença dos seus derivados nas placas ateroscleróticas constitui indicativo dessa contribuição.

Dentre os principais efeitos associados aos OsC também estão as alterações das propriedades das membranas celulares, que afetam as funções básicas das mesmas (transporte de nutrientes, atividade de enzimas da membrana e equilíbrio osmótico), a mutagenicidade e a carcinogenicidade (BÖSINGER, LUF e BRANDL, 1993).

Correlação positiva entre a formação de 5,6-epoxicolesterol e a incidência de câncer foi evidenciada em experimentos por meio do desenvolvimento de células cancerígenas em ovário e pele de ratos (BLACK e DOUGLAS, 1972). Esse efeito mutagênico do 5,6-epoxicolesterol também foi demonstrado em pesquisas in vitro (SEVANIAN e PETERSON, 1984).

A relação dos $\mathrm{Os} C$ com processos cancerígenos foi sugerida ainda em estudos epidemiológicos para pacientes com níveis elevados de Triol no trato gastrointestinal (SEVANIAN e PETERSON, 1984).

\section{CONCLUSÃO}

Estudos sobre OsC em pescado são escassos na literatura e os resultados, às vezes, conflitantes. Mas foi possível concluir que as condições inadequadas de processamento e armazenamento a que o pescado é submetido aumentam o seu potencial para a formação de OsC. Entre os óxidos formados, o $7 \alpha-\mathrm{OH}, 7 \beta-\mathrm{OH}$ e o 7 -Ceto são os de maior predominância. Os óxidos mais citotóxicos e aterogênicos, o 25-OH e o Triol, são encontrados em algumas espécies.

A descoberta de novos óxidos em peixes, como 19-hidroxicolesterol, 22 "R"-hidroxicolesterol, 22 "S"-hidroxicolesterol, 24 "S"-hidroxicolesterol e 25 "R"-hidroxicolesterol, sugere a existência de OsC ainda não reportados para pescado.

Enquanto a oxidação do colesterol em pescado não for devidamente estudada, a potencialidade desses produtos para a formação de OsC continuará sendo mal estimada e favorecida pela falta de procedimentos tecnológicos adequados. Pesquisas são também necessárias para que quantidades inócuas de consumo possam ser estabelecidas.

\section{ABSTRACT}

\section{OXIDATION OF CHOLESTEROL IN FISH}

The fish presents in their lipid fraction fatty acids with a high degree of unsaturation together with elevated levels of cholesterol in some species. This factor, is associate with technological procedures, favour the oxidation of cholesterol. Cholesterol oxides are biologically active and are capable of inducing 
cytotoxic, atherogenic, mutagenic and carcinogenic processes. For these reasons, interest in oxidation phenomena has increased considerably. This review paper examined aspects related to cholesterol oxides, their formation in foods and their biological effects, with particular emphasis on the formation of cholesterol oxides in fish. The study revealed that the most common cholesterol oxides found in food, including the most cytotoxic and atherogenic, are also present in these products.

KEY-WORDS: CHOLESTEROL; OXIDATION; CHOLESTEROL OXIDES; FISH.

\section{REFERÊNCIAS}

1 BADOLATO, E.S.G.; CARVALHO, J.B.; AMARAL MELLO, M.R.P.; TAVARES, M.; CAMPOS, N.C.; AUED-PIMENTEL, S.; MORAIS, C. Composição centesimal de ácidos graxos e valor calórico de cinco espécies de peixes marinhos nas diferentes estações do ano. Rev. Inst. Adolfo Lutz, v. 54, n.1, p. 27-35, 1994.

2 BAGGIO, S.R. Óxidos de colesterol, lipídios totais e ácidos graxos em produtos cárneos processados. Campinas, 2004. 215 p. Tese (Doutorado em Ciência dos alimentos), Faculdade de Engenharia de Alimentos, Universidade Estadual de Campinas.

3 BLACK, H.S.; DOUGLAS, D.R. A model system for the evaluation of the role of cholesterol $\alpha$-oxide in ultraviolet carcinogenesis. Cancer Res., v. 32, n.12, p. 2630-32, 1972.

4 BÖSINGER, S.; LUF, W.; BRANDL, E. Oxysterols: their occurrence and biological effects. Int. Dairy J., v. 3, p.1-33, 1993.

5 CARERI, M.; FERRETTI, D.; MANINI, P.; MUSCI, M. Evaluation of particle beam high-performance liquid chromatographymass spectrometry for analysis of cholesterol oxides. J. Chrom., v. 794, n.1-2, p. 253-62, 1998.

6 CHEN, B.H.; CHEN, Y.C. Evaluation of the analysis of cholesterol oxides by liquid chromatography. J. Chromatogr. A., v. 661, n. $1-2$, p.127-36, 1994.

7 CHEN, Y.C.; CHIU, C.P.; CHEN, B.H. Determination of cholesterol oxides in heated lard by liquid chromatography. Food Chem., v. 50, n.1, p. 53-58, 1994.

8 CHEN, J.T.; WANG, H.C.; CHEN, B.H. Kinetic model of the cholesterol oxidation during heating. J. Agric. Food Chem., v. 46, n. 7 , p. $2572-77,1998$

9 CHEN, J.; YEH, G. Cholesterol oxidation products in small sun-dried fish. Food Chem., v.50, n. 2, p.167-70, 1994.

10 CONN, E.E.; STUMPF, P.K. Introdução à bioquímica. 3. ed. São Paulo: Edgard Blücher, 1975. 447 p.

11 CRASTES DE PAULET, A.; DOUSTE-BLAZY, L.; PAOLETTI, R. (eds). Free radicals, lipoproteins membrane lipids. New York: Plenum Press, 1990. p.115-32.

12 ECHARTE, M.; CONCHILLO, A.; ANSORENA, D.; ASTIASARÁN, I. Óxidos de colesterol em langostinos frescos y congelados, crudos y a la plancha. Nutr. Hosp., v. 20, n. 4, p. 293-96, 2005.

13 ECHARTE, M.; ZULET, M.A.; ASTIASARÁN, I. Oxidation process affecting fatty acids and cholesterol in fried and roasted salmon. J. Agric. Food Chem., v. 49, n.11, p. 5662-67, 2001.

14 GUARDIOLA, F.; BOU, R.; BOATELLA, J.; CODONY, R. Analysis of sterol oxidation products in foods. J. AOAC Int., v. 87, n. 2, p. 441-66, 2004.

15 GUARDIOLA, F.; CODONY, R.; RAFECAS, M.; BOATELLA, J. Comparison of three methods for the determination of oxysterols in spray-dried egg. J. Chromatogr., v. 705, n. 2, p. 289-304, 1995a.

16 GUARDIOLA, J.; CODONY, R.; RAFECAS, M.; BOATELLA, J. Formación de derivados oxidados del colesterol en alimentos. Grasas y Aceites, v. 46, n. 3, p. 202-12, 1995b.

17 GUTTERIDGE, J.M.C.; HALLIWELL, B. Antioxidants in nutrition, health, and disease. New York: Oxford University Press, 1994. p.63-81.

18 HARPER, H.A.; RODWELL, W.M.; MAYES, P.A. Metabolismo dos lipídeos. 1. Ácidos graxos. MANUAL de Química Fisiológica. 5. ed. São Paulo: Atheneu, 1982. p. 334-56.

19 HOFFMANN, G. The chemistry and technology of edible oil fats and their high fat products. San Diego, C. A.: Academic Press, 1989. p.14-15.

20 JOHNSTON, J.J.; GHANBARI, H.A.; WHEELER, W.B.; KIRK, J.R. Characterization of shrimp lipids. J. Food Sci., v. 48, n.1, p. 33-35, 1983. 
21 KAO, Y.M.; HWANG-SUN, L. Analysis of cholesterol oxidation products in dried squid. Food Sci., v. 24, n. 2, p. 242$57,1997$.

22 KORAHANI, V.; BASCOUL, J.; CRASTED DE PAULET, A. Autoxidation of cholesterol fatty acid esters in solid states and aqueous dispersion. Lipids, v. 17, n.10, p. 703-08, 1982.

23 LI, S.X.; CHERIAN, G.; AHN, D.U.; HARDIN, R.T.; SIM, J.S. Storage, heating and tocopherols affect cholesterol oxides formation in food oils. J. Agric. Food Chem., v. 44, n.12, p.3830-34, 1996.

24 LUZIA, L.A. Influência da sazonalidade no valor nutricional e perfil lipídico em cinco espécies populares de pescado. São Paulo, 2000. 104 p. Dissertação (Mestrado em Nutrição), Faculdade de Saúde Pública, Universidade de São Paulo.

25 MAERKER, G. Cholesterol autoxidation-current status. J. Am. Oil Chem. Soc., v. 64, n. 3, p. 388-92, 1987.

26 MASORO, E.D. Physiological chemistry of lipids in mammals. Philadelphia: W. B. Saunders Company, 1968. cap.7, p. 96.

27 McCLUSKEY, S.; DEVERY, R. Validation of chromatography analysis of cholesterol oxides in dried foods. Trends Food Sci. Technol., v.4, n.6, p.175-78, 1993.

28 MORALES-AIZPURÚA, I.C.; TENUTA-FILHO, A. Colesterol, 7-cetocolesterol e 25-hidroxicolesterol em maionese. Ciênc. Tecnol. Aliment., v. 25, n. 3, p. 495-99, 2005

29 MORALES-AIZPURÚA, I.C.; TENUTA-FILHO, A. Óxidos de colesterol: ocorrência em alimentos, formação e efeitos biológicos. Rev. Bras. Ciênc. Farm., v. 38, n. 4, p. 431-42, 2002.

30 MORGAN, J.N.; ARMSTRONG, D.J. Quantification of oxidation products in egg yolk powder spray-dried with direct heating. J. Food Sci., v. 57, n.1, p. 43-45, 1992.

31 MOURA, A.F.P. Efeito do processamento térmico sobre a ocorrência do 7-cetocolesterol em camarãorosa (Penaeus brasiliensis + Penaeus paulensis). São Paulo, 1999. 204 p. Dissertação (Mestrado em Bromatologia), Faculdade de Ciências Farmacêuticas - Universidade de São Paulo.

32 MOURA, A.F.P.; TENUTA-FILHO, A. Efeito do processamento sobre os níveis de colesterol e 7-cetocolesterol em camarão-rosa. Ciênc. Tecnol. Aliment., v. 22, n. 2, p.117-21, 2002.

33 MUSTAFA, F.A.; MEDEIROS, D.M. Proximate composition, mineral content, and fatty acids of catfish (Icatalarus puntactus, Rafinesque) for different seasons and cooking methods. J. Food Sci., v. 50, n. 3, p. 585-88, 1985.

34 NAWAR, W.W. Lipids. apud FENNEMA O.R. Food Chem. $3^{\text {rd }}$ ed. New York: Marcel Dekker, 1996. p. $225-320$.

35 NIELSEN, J.H.; OLSEN, C.E.; JENSEN, C.; SKIBSTED, L.H. Cholesterol oxidation in butter and dairy spread during storage. J. Dairy Res., v. 63, n.1, p.159-67, 1996.

36 OHSHIMA, T.; LI, N.; KOIZUMI, C. Oxidative decomposition of cholesterol in fish products. J. Am. Oil Chem. Soc., v.70, n. 6, p. 595-99, 1993.

37 OHSHIMA, T.; SHOZEN, K.; USHIO, H.; KOIZUMI, C. Effects of grilling on formation of cholesterol oxides in seafoods products rich in polyunsaturated fatty acids. Lebensm. Winss. Technol., v. 70, n. 6, p. 595-99, 1996.

38 OSADA, K.; KODAMA, T.; YAMADA, K.; SUGANO, M. Oxidation of cholesterol by heating. J. Agric. Food Chem., v. 41, n. 8, p.1198-202, 1993.

39 PANIANGVAIT, P.; KING, A.J.; JONES, A.D.; GERMAN, B.G. A critical review cholesterol oxides in foods of animal origin. J. Food Sci., v. 60, n. 6, p.1159-74, 1995.

40 PARDI, M.C.; SANTOS, L.F.; SOUZA, E.R.; PARDI, H.S. Ciência, higiene e tecnologia da carne. Goiânia: UFG, 2001. $623 \mathrm{p}$.

41 PENG, S.; HU, B.; MORIN, R.J. Angiotoxicity and atherogenicity of cholesterol oxides. J. Clin. Lab. Anal., v. 5, n. 2, p. 144-52, 1991.

42 PENG, S.K.; THAM, P.; TAYLOR, C.B.; MIKKELSON, B. Cytotoxicity of cholesterol oxidation derivates on cultured aortic smooth muscle cells and their effect on cholesterol biosynthesis. Am. J. Clin. Nutr., v. 32, n. 5, p.1033-42, 1979.

43 PINCINATO, E.D.C. Aterogenicidade dos óxidos de colesterol: estudo da esterificação mediada pela LCAt e da transferência entre lipoproteínas. São Paulo, 2000. 83 p. Dissertação (Mestrado em Bioquímica), Faculdade de Ciências Farmacêuticas - Universidade de São Paulo.

44 RODRIGUEZ-ESTRADA, M.T.; PENAZZI, G.; CABONI, M.F.; BERTACCO, G.; LERCKER, G. Effect of different cooking methods on some lipid and protein components of hamburgers. Meat Sci., v. 45, n. 3, p. 365-75, 1997. 
45 SALDANHA, T.; SAWAYA, A.C.H.F.; EBERLIN, M.N.; BRAGAGNOLO, N. HPLC separation and determination of 12 cholesterol oxidation products in fish: comparative study of RI, UV, and APCI-MS detectors. J. Agric. Food Chem., v. 54, n.12, p. $4107-13,2006$.

46 SAMPAIO, G.R. Ocorrência de óxidos de colesterol e análise do perfil lipídico em camarão salgado-seco. São Paulo, 2004. 93 p. Dissertação (Mestrado em Nutrição Humana Aplicada), Universidade de São Paulo.

47 SANCHEZ, L. Pescado: matéria-prima e processamento. Campinas: Cargil, 1989. p.1-14.

48 SEBEDIO, J.L.; RATNAYAKE, W.M.N.; ACKMAN, R.G.; PREVOST, J. Stability of polyunsaturated É-3 fatty acids during deep fat frying of Atlantic mackerel (Scomber scombrus L.). Food Res. International, v. 26, n. 3, p.163-72, 1993.

49 SEVANIAN, A.; PETERSON, A.R. Cholesterol epoxide is a direct acting mutagen. Proc. Nat. Acad. Sci., v. 81, n.13, p. 4198-202, 1984.

50 SHOZEN, K.; OHSHIMA, T.; USHIO, H.; KOIZUMI, C. Formation of cholesterol oxides in marine fish products induced by grilling. Fish Sci., v. 61, n. 5, p. 817-21, 1995.

51 SILVA, F.A.M.; BORGES, M.F.M.; FERREIRA, M. Métodos para avaliação do grau de oxidação lipídica e da capacidade antioxidante. Quim. Nova, v. 22, n.1, p. 94-103, 1999.

52 SMITH, L.L. Analysis of oxysterols by liquid chromatography. J. Liq. Chrom., v.16, n. 8, p. 1731-47, 1993.

53 SMITH, L.L. Cholesterol autoxidation 1981-1986. Chem. Phys. Lipids, v. 44, n. 2-4, p. 87-125, 1987.

54 SMITH, L.L. Review of progress in sterol oxidation: 1987-1995. Lipids, v. 31, n. 5, p. 453-87, 1996.

55 TAI, C.Y.; CHEN, Y.C.; CHEN, B.H. Analysis, formation and inhibition of cholesterol oxidation products in food: an overview (Part I). J. Food Drug Anal., v. 7, n. 4, p. 243-57, 1999.

56 TAYLOR, C.B.; PENG, S.K.; WERTHESSEN, N.T.; THAM, P.; LEE, K.T. Spontaneously occurring angiotoxic derivates of cholesterol. Am. J. Clin. Nutr., v. 32, n.1, p. 40-57, 1979.

57 TSAI, L.S.; IJICHI, K.; HUDSON, C.A; MEEHAN, J.J. A method for the quantitative estimations of cholesterol $\alpha$-ocide in eggs. Lipids, v. 15, n. 3, p.124-28, 1980.

58 VALENZUELA, A.B.; SANHUEZA, J.C.; NIETO, S.K. Oxidos del colesterol (oxisteroles): factores que condicionan su formacion, efectos biologicos, y su presencia en los alimentos. Rev. Chil. Nutr., v. 29, n. 2, p. 116-24, 2002.

59 ZUNIN, P.; BOGGIA, R.; EVANGELISTI, F. Identification and quantification of cholesterol oxidation products in canned tuna. J. Am. Oil Chem. Soc., v. 78, n.10, p.1037-40, 2001. 